Recepción: 18/ 07/ 2018

Aceptación: 12 / 09 / 2018

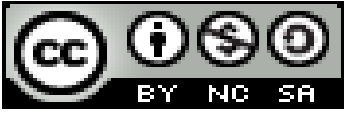

Publicación: 05 / 10 / 2018

\title{
Las tecnologías de la información y la comunicación en el proceso de enseñanza de la música
}
The technologies of information and communication in the music teaching process

\section{As tecnologias da informação e da comunicação no processo de ensino da música}

Oscar E. Bolívar- Chávez ${ }^{\mathrm{I}}$

obolivar@utm.edu.ec

Yury V. Palma II

ypalma@utm.edu.ec

Johanna Zambrano-Sornoza III

jmzambranos@utm.edu.ec
Ramón Toala-Dueñas ${ }^{\text {IV }}$

rtoala@utm.edu.ec

José G. Pico-Mieles V

jgpico@edu.ec

\section{Correspondencia: obolivar@utm.edu.ec}

I Máster en Nuevas Tecnologías Aplicadas a la Educación, Licenciado en Ciencias de la Educación Especialidad Instrumentista Pedagogo en Saxofón, Docente Universidad Técnica de Manabí, Portoviejo, Ecuador.

II Docente Universidad Técnica de Manabí, Portoviejo, Ecuador.

III Magíster en Gerencia Educativa, Licenciada en Ciencias de la Educación Técnica Mención Contabilidad Computarizada, Docente Universidad Técnica de Manabí, Portoviejo, Ecuador.

IV Magíster en Informática Empresarial, Ingeniero en Sistemas Informáticos, Tecnólogo Programador en Computación, Docente Universidad Técnica de Manabí, Portoviejo, Ecuador.

v Tecnólogo Programador en Computación, Licenciado en Ciencias de la Educación Especialidad Historia y Geografía, Docente Universidad Técnica de Manabí, Portoviejo, Ecuador. 


\title{
Resumen
}

Aunque existen numerosos trabajos sobre las tecnologías de la información y la comunicación como recurso didáctico en el aula, cambiar la dirección y enfatizar la problemática que existe en el uso y adaptación de las posibilidades abiertas por el avance tecnológico de las redes, hace necesario revisar los diseños de aplicaciones, que aunque no están concebidas para la enseñanza de la música, sí pueden tener una aplicación práctica, pues muchas de ellas pueden conectarse con estándares de aprendizaje para el desarrollo de dicha enseñanza. Con base a lo expuesto, se expone en este artículo un análisis de uso de las tecnologías de la información y la comunicación en el proceso de enseñanza de la música. La metodología fue de tipo descriptivo -documental y el corpus de la investigación la constituyeron la revisión de fuentes bibliográficas de tipo académicas o de divulgación en formato físico o digital. El análisis hermenéutico de los contenidos analizados, permitió concluir que se hace impostergable la introducción de las TIC en el contexto educativo como un recurso pedagógico de apoyo en la enseñanza- aprendizaje de la música.

Palabras clave: tecnología de la información y comunicación; música; enseñanza -aprendizaje; avance tecnológico.

\begin{abstract}
Although there are numerous works on information and communication technologies as a didactic resource in the classroom, changing the direction and emphasizing the problems that exist in the use and adaptation of the possibilities opened by the technological advance of the networks, it is necessary to review the applications designs, that although they are not conceived for the teaching of music, they can have a practical application, since many of them can be connected with learning standards for the development of said teaching. Based on the above, this article presents an analysis of the use of information and communication technologies in the process of teaching music. The methodology was of descriptive type -documental and the corpus of the investigation constituted the revision of bibliographic sources of academic type or of dissemination in physical or digital format. The hermeneutic analysis of the contents analyzed allowed us to conclude that the introduction of ICT in the educational context as a pedagogical resource of support in the teaching-learning of music becomes urgent.
\end{abstract}


Key words: information technology and communication; music; teaching learning; technological advance.

\section{Resumo}

Embora existam numerosas obras sobre tecnologia da informação e comunicação como recurso didático em sala de aula, mudar de direção e enfatizar os problemas que existem no uso e adaptação das possibilidades abertas pelo avanço tecnológico das redes torna necessário rever a projetos de aplicativos, que embora não sejam concebidos para o ensino de música, eles podem ter uma aplicação prática, uma vez que muitos deles podem ser conectados com padrões de aprendizagem para o desenvolvimento do referido ensino. Com base no exposto, este artigo apresenta uma análise do uso das tecnologias de informação e comunicação no processo de ensino de música. A metodologia foi de tipo descritiva - documental e o corpus da investigação constituiu a revisão de fontes bibliográficas de tipo acadêmico ou de divulgação em formato físico ou digital. A análise hermenêutica dos conteúdos analisados permitiu concluir que a introdução das TIC no contexto educacional como recurso pedagógico de apoio no ensinoaprendizagem da música torna-se urgente.

Palavras chave: tecnologia da informação e comunicação; música; ensino-aprendizagem; avanço tecnológico.

\section{Introducción}

En el contexto educativo, las TIC se han ido implantando y desarrollando en los últimos años. Grandes empresas como Google o Microsoft están creando plataformas específicas para su aplicación en el campo educativo, condicionadas al uso de Internet. Recientemente, Google ha abierto al público su plataforma Google Classroom. Microsoft también ha desarrollado una plataforma de aprendizaje virtual online a través de Office 365 y ha adaptado su sistema operativo al entorno educativo mediante el lanzamiento de Windows 10S, en el que junto a otras herramientas se incluye una versión adaptada del videojuego Minecraft para entornos educativos.

La llamada revolución digital, es el conjunto de innovaciones tecnológicas que han hecho posible que la información (sonido, imagen, texto) se digitalice, es decir, que se transmita a gran velocidad gracias a la simplificación de estos datos a combinaciones de ceros y unos integradas 
dentro de un circuito electrónico. Estos avances tecnológicos convergieron todos sobre la misma materia prima, la información, así como en la creación y el uso de redes de comunicaciones. En este contexto, Internet se convirtió en el foco de esta convergencia, y desde que Internet se ha implantado en nuestras vidas, el uso de las Tecnologías de la Información y Comunicación (TIC) se ha extendido a un amplio abanico de actividades relacionadas con la cultura, el ocio, las cuestiones domésticas, la comunicación con otras personas y, por supuesto a la educación. El conocimiento y manejo instrumental de estas tecnologías, la forma de interpretar o de relacionarse con la realidad a través de ellas y las implicaciones sociales que todo esto conlleva ya forman parte de la cultura de nuestro tiempo.

No hay duda de que, las innovaciones educativas están adquiriendo un papel fundamental en el sector de la educación. Todas ellas coinciden en el uso de Internet como soporte principal, lo que ha favorecido sobre todo el desarrollo de entornos personales de aprendizaje. En el área de la música, prácticamente no existen innovaciones y las pocas que son susceptibles de ser utilizadas no están diseñadas específicamente para esta materia. Son aplicaciones concebidas fuera del ámbito de la educación, muchas de ellas profesionales, lo cual limita su utilización. Esto genera dificultades por la necesidad de formación de los docentes, el elevado precio de las licencias, etc. Además, muchas de las TIC existentes se utilizan de forma que no se aprovecha su amplio potencial. Se sigue utilizando el aula de informática principalmente para acceder a los contenidos informativos que proporciona la red, o se usa la pizarra digital como un simple proyector y no se produce ningún tipo de innovación educativa.

Por otro lado, desde hace unos años hasta ahora se ha dotado a las aulas de ordenadores, proyectores y pizarras digitales, creyendo que todos los docentes van a hacer un buen uso de ellos. Se cree que facilitaría enseñar a los alumnos gracias a las Nuevas Tecnologías, pero, de nada sirve modernizar las escuelas sin modernizar la metodología. Las Tecnologías de la Información y la comunicación (TIC) pueden transformar la enseñanza tradicional, pero esto sólo se conseguirá si los docentes reflexionan sobre qué y cómo se enseña. Esta posición, devela la necesidad de analizar el uso de las tecnologías de la información y la comunicación en el proceso de enseñanza de la música. 


\section{Desarrollo}

El desarrollo tecnológico afecta ya a todas las áreas de la vida y los miembros de la comunidad, y en el futuro inmediato es previsible que lo haga aún más. Nuestro alumnado como ciudadanos del siglo XXI tienen unas necesidades, unas motivaciones y una actitud hacia las TIC completamente distintas que el de hace quince años. Es cierto que en la actualidad los centros educativos se muestran razonablemente eficaces en los procesos de socialización, integración, educación afectiva, formación ética y en valores. Sin embargo, los procesos, los materiales y las herramientas de aprendizaje aún se pueden mejorar para adaptarse a esta nueva situación. Por tanto, la escuela necesita actualizar su metodología para afrontar este gran reto.

En este sentido, Área (1998) comenta que:

"la socialización cultural de los niños y niñas cada vez en mayor medida se produce a través de la utilización de distintas tecnologías de la información que utilizan prácticamente desde que nacen (televisión, vídeo, videojuegos, móvil, Internet...). Los actuales ciudadanos menores de diez años son, en este sentido, la primera generación nacida y amamantada culturalmente en la llamada sociedad de la información. En consecuencia, esta nueva generación cada vez aprende más cosas fuera de la escuela a través del uso de las distintas tecnologías audiovisuales e informáticas. Dicho de otro modo, cada día los jóvenes acceden a más educación fuera del contexto escolar a través de soportes multimedia, de software didáctico, de televisión digital, de redes informáticas, de programas audiovisuales para vídeo...”. (pag.47)

La aplicación de las tecnologías de la información y la comunicación en el proceso de enseñanza de la Música tiene muchas posibilidades. Los ordenadores se han convertido hoy por hoy en una herramienta poderosa a disposición del profesorado de Música en el proceso de enseñanzaaprendizaje. El mismo autor, plantea que entre los requisitos, necesidades y posibilidades que requieren los tics en la enseñanza de la música están la infraestructura, el equipamiento y la conectividad.

\section{Infraestructura}

La infraestructura debe disponer de aulas con un equipamiento informático básico para uso por parte del alumnado. Lo habitual es que el grueso de este equipamiento esté ubicado en el Aula de 
Informática. No obstante, cada vez son más numerosas las dotaciones realizadas bajo el criterio de distribuir los dispositivos de forma generalizada con el fin de se conviertan en un elemento más dentro de las aulas. En este momento podemos encontrarnos con distintas situaciones que condicionan la forma en que se puede planificar el trabajo con las TIC dentro de la materia de Música.

\section{El equipamiento}

El hardware o equipamiento físico que se requiere dentro del aula de música no tiene por qué ser demasiado costoso. Un ordenador personal que cuente con las características básicas que están disponibles hoy en día en un equipo de tipo medio será suficiente para satisfacer los requerimientos mínimos. En cuanto a los periféricos necesarios (dispositivos que permiten al ordenador interactuar con el exterior por medio de la entrada, salida o almacenamiento de datos, así como la comunicación entre distintos ordenadores) además de los habituales de cualquier equipo (teclado alfanumérico, ratón, monitor, impresora, MODEM...), uno o varios micrófonos, y tarjetas de sonido y altavoces o auriculares suficientes para cada equipo y/o alumno, según el caso. Para la materia de música contar con este tipo de elementos es imprescindible, pero, además, es necesario que posean una mínima calidad y funcionen perfectamente. Las razones son evidentes ya que su uso didáctico en la música será habitual tanto grabar sonidos como reproducirlos a través del ordenador.

\section{La conectividad}

La conectividad hace referencia, de forma genérica, a la capacidad de los dispositivos de estar conectados entre sí y comunicarse a través de sistemas de red. En todos los centros debe existir conectividad entre los equipos informáticos a través de una red de área local que además ofrezca salida a Internet, que garantice acceso a los alumnos y alumnas a la Red de una forma eficaz y óptima, pues para todas aquellas actividades que contemplen la utilización de información, sistemas de comunicación o aplicaciones en línea es imprescindible.

No obstante, para llevar a cabo muchas de las propuestas didácticas con TIC en el área de Música no serán imprescindible. En unos casos porque el enfoque no lo hará preciso; en otros porque será conveniente descargar los recursos previamente y replicarlos en los ordenadores que utilicen los alumnos o servirlos a través de la Intranet del centro escolar. En cualquier caso, siempre será muy 
recomendable. El tipo de conectividad de la que dispongamos en el centro: alámbrica o inalámbrica (Wi-Fi, WiMAX...) unido a la distribución que haya del equipamiento (en salas específicas, distribuida, pizarras electrónicas, portátiles con cañón...) condicionará también las posibilidades que se tendrá a la hora de planificar sesiones de trabajo utilizando las TIC, por lo que es un elemento a considerar. También deben estimarse, otras opciones de comunicación inalámbrica (Bluetooth...) que posea el equipamiento, así como los dispositivos portátiles de los alumnos y alumnas (iPod, reproductores de audio digital, PDA.) pues ello facilita la transferencia habitual y cotidiana de información entre unos y otros. Entorno MIDI.

Dentro del ámbito musical, el protocolo MIDI (Musical Instrument Digital Interface) es una forma específica de conectividad que posee, además, otras muchas implicaciones ya que es capaz de transmitir instrucciones musicales entre un ordenador y cualquier equipo MIDI, o entre dos o varios dispositivos MIDI. Un archivo MIDI contiene las instrucciones necesarias para reproducir una pieza musical. La información que maneja el protocolo MIDI es digital (secuencias de ceros y unos) y no transfiere sonido si no solo instrucciones. Esto quiere decir que lo que permite es que un equipo le indique a otro cuándo comienza a sonar, qué nota debe hacerlo, durante cuánto tiempo, etc., pero la reproducción del sonido siempre depende del dispositivo que recibe la información. (http://www.slideshare.net/AnaBasterra/los-proyectosde-aprendizaje)

\section{Incidencia de las TIC en la educación musical}

Actualmente, el empleo de las herramientas TIC en actividades de enseñanza y aprendizaje de Música, obedece, muchas veces, a actitudes e iniciativas personales de algunos docentes. De todos depende que esta situación se generalice y permita que sus ventajas lleguen al mayor número posible de miembros de la comunidad educativa. Entendiendo, que las TICs potencian modelos pedagógicos renovados en los que el alumnado toma mayor protagonismo de su aprendizaje.

La introducción de las TIC en el contexto educativo pasa necesariamente porque el profesorado tenga la formación necesaria para poder incorporarlas en el aula. Es decir, de la misma manera que usamos el instrumental Orff para acompañar una melodía de flautas o visionamos un fragmento de la película Amadeus para explicar las precoces capacidades interpretativas de Mozart, podríamos desarrollar nuestra programación integrando determinadas actividades de 
aprendizaje on line. Lo extraordinario de estos nuevos recursos es que permiten imaginar numerosos tipos de aplicaciones didácticas, pues son un terreno abonado a la innovación. Aunado, a la aparición de nuevos modos de comunicación sobre todos entre los más jóvenes, utilizando para ello aplicaciones de mensajería instantánea, chats, blogs, redes sociales. (Barco, 2011)

Asimismo, el profesorado de educación musical frente a las TIC "Igual que es necesario mojarse si uno quiere aprender a nadar, el profesorado que pretenda integrar provechosamente las tecnologías en su aula de Educación Musical es necesario que se implique, manejando las tecnologías y elaborando materiales musicales digitales (...) y para ello no existe otra alternativa que invertir tiempo, formación y esfuerzo". (Torres, 2010, p.27).

\section{Recursos informáticos relacionados con la Educación Musical}

Aparte de los programas de Software libre, existen otros recursos informáticos que pueden emplearse en el aula de música y resultan muy beneficiosos para favorecer la educación, entre estos las Web o Blog de los docentes, pizarra digital interactiva La Pizarra Digital Interactiva (PDI) y los Scratch.

\section{Las Web o Blog de los docentes.}

Una Web es una página de Internet donde se refleja un trabajo sobre un tema. Se considera algo vivo que se va actualizando en un breve período de tiempo. Sirve para reflexionar contenidos o pensamientos con el fin de difundirlos para darlos a conocer. Pavón, (2013), indica "Es un importante elemento de orientación para los alumnos y facilitan el contacto con otros profesores e investigadores". (p.42). Además, se puede compartir la creación de materiales y recursos educativos e incluir apuntes. Por otro lado, los juegos de música en las web constituyen una herramienta excelente para el aula de música. La diversión es importante en el aprendizaje y existen una gran variedad de juegos de música en Internet para tratar diferentes contenidos musicales. En este sentido, Camino (2010), señala que "Si tenemos preparado un buen surtido de pasatiempos musicales conseguiremos unir aprendizaje y diversión sin problemas. Otro aspecto a tener en cuenta para programar los juegos musicales es la facilidad para realizarlos en grupo utilizando la pizarra digital interactiva que tanto entusiasma a nuestros alumnos". (p.1). 


\section{Pizarra digital interactiva La Pizarra Digital Interactiva (PDI).}

Según Torres (2010), es un sistema tecnológico que necesita un ordenador con conexión a Internet, un proyector de vídeo y una superficie táctil donde se proyecte la imagen de la pantalla del ordenador, permitiendo interactuar sobre ella. Las pizarras digitales permiten escribir sobre ella y controlar programas informáticos, incluidas las aplicaciones de Internet, con un puntero o con el dedo. El mismo autor, sostiene que "lo más importante no es la pizarra, sino las actividades que se desarrollen en ella" (pag.196). Es de notar, que tener numerosos y variados recursos tecnológicos en el aula de música, si no existe preparación por parte del docente para llevarlos a cabo y si no se emplean con unos objetivos didácticos claros, el uso de las TIC perderá una importante parte de su finalidad.

\section{Scratch.}

Un nuevo recurso para la Educación Musical, según Wing (2006) es el pensamiento computacional, entendiendo que es como una habilidad fundamental para todos y no de uso exclusivo para los informáticos. Además, el pensamiento computacional implica resolver problemas, diseñar sistemas y comprender el comportamiento humano, basándose en los conceptos fundamentales de la informática. Explica que el Scratch permite trabajar el pensamiento computacional en el aula de música, dado que es un entorno de aprendizaje de lenguaje de programación que permite a los principiantes obtener resultados sin tener conocimientos formales básicos y facilitando una instrucción autónoma. Está pensado para desarrollar juegos, animaciones y creaciones multimedia en general mediante la colocación de bloques de acciones. Este programa incorpora una serie de bloques que permite realizar trabajos musicales muy interesantes, facilitando la expresión del alumnado de forma creativa.

\section{Fuentes y Metodología}

La metodología fue de tipo descriptivo y documental. Según Arias (2012), la investigación descriptiva se caracteriza por describir la estructura o comportamiento de la variable estudiada tal como se presenta, en este caso particular se analizó la situación actual de las TIC aplicadas al proceso de enseñanza y uso de la música. Asimismo, permitió el registro, análisis e interpretación de las teorías manejadas en el estudio. El corpus de la investigación la constituyó la revisión de fuentes bibliográficas y no bibliográficas y el enfoque epistémico fue de tipo hermenéutico, 
permitiendo el análisis de los contenidos de la información consultada de tipo académica o de divulgación, en formato físico o digital.

\section{A manera de conclusiones}

La introducción de las TIC en el contexto educativo se ha convertido en una necesidad acuciante. La mayor parte del profesorado dispone de ordenador personal y de conexión a Internet en su domicilio. Aun cuando actualmente la existencia y uso de una gran cantidad de actividades tecnológicas, desde asuntos personales hasta de tipo comunitario. Sin embargo, es notorio una obsolescencia en el patrón de enseñanza, donde el docente es el principal dador de información desde los textos, la pizarra pautada, partituras adaptadas a instrumental Orff y audiciones en CD.

De allí que el avance de la tecnología, exige a los docentes aceptar que ha dejado de ser el único vehículo de transmisión e interpretación de la información. Ahora es fundamental saber orientar al alumnado sobre cómo acceder a la información y cómo ésta debe procesarse. Interaccionar con el alumnado para ayudarle a elaborar conocimiento significativo, es decir, que sean capaces de buscar y transformar la información en conocimiento que les sea útil, comprensible y sean capaces de transferirla durante el proceso de enseñanza aprendizaje, específicamente del área de música a través del uso de las bases de datos, software, enciclopedias en línea, programas educativos multimedia, buscadores, blogs y podcasts, entre otras. Se trata de enseñar a los estudiantes a aprender, y ello exige que el profesor, lejos de proponer una serie de actividades iguales para todos, facilite al alumnado distintos itinerarios, actividades y medios que resulten acordes a sus capacidades y estilos cognitivos. Por lo tanto, con este tipo de herramientas el profesorado puede personalizar los recursos para que se adecuen a los diferentes estilos de aprendizajes.

También la mayoría de los docentes, que no son nativos digitales, deben dominar el uso de las TIC para poder utilizarlas como herramientas útiles en su práctica educativa, así como para reducir la brecha existente entre la realidad de la sociedad y las aulas. Entendiendo que la importancia de la formación del profesorado en las tecnologías es fundamental para lograr un éxito en la integración de las TIC a la Educación Musical. A pesar de que los maestros de Educación Musical siempre han estado más ligados a las nuevas tecnologías con el uso de equipos de música, reproductores de vídeo, grabadoras o teclados electrónicos, las TIC no se han 
introducido de la misma manera si nos referimos al proceso enseñanza-aprendizaje. La utilización de las TIC en el aula de música es más reducida que en otras áreas de conocimiento.

\section{Referencias Bibliográficas}

Área, M. (1998) ¿Qué aporta internet al cambio pedagógico? Recuperado en:

https://www.google.co.ve/search?q=Manuel+\%C3\%81rea+(1998)\&oq=Manuel+\%C3\%81rea+(1 998)\&aqs=chrome...69i57.1519j0j7\&sourceid=chrome\&ie=UTF-8

Arias, F. (2012). El proyecto de investigación. Introducción a la metodología científica. 6ta. Edición. Venezuela. Ediciones Fidias.

Barco, M. (2014). Evolución de las TIC. Recuperado en: http://www.slideshare.net/MJBARCO/evolucin-tic.

Basterra, A. (2014). Los proyectos de aprendizaje. Recuperado en: http://www.slideshare.net/AnaBasterra/los-proyectosde-aprendizaje-34451926.

Camino, M. (2010). Juegos Musicales, un mundo de aprendizaje y diversión. Educacontic.es. Recuperado en: http://www.educacontic.es/va/blog/juegos-musicales-un-mundo-de-aprendizajeydiversion

Pavón, F. (2013). La introducción de las TIC en el currículum y en la organización escolar de la educación infantil y primaria. En M ${ }^{\text {a }}$. C. Martínez Serrano (Coord.), Buenas prácticas educativas en el uso de las TIC. (pp. 11-50). Jaén: Joxman.

Torres, L. (2010). Las TIC en el aula de educación musical. Bases metodológicas y posibilidades prácticas. Sevilla: MAD, eduforma.

Wing, J. M. (2006). Computational thinking. Viewpoint, 49 (3), 33-35. 